\title{
Asynchronous and Tailored Digital Rehabilitation of Chronic Shoulder Pain: A Prospective Longitudinal Cohort Study
}

\author{
Dora Janela (iD) \\ Fabíola Costa (D) \\ Maria Molinos (iD) \\ Robert G Moulder ${ }^{2}$ \\ Jorge Lains (D) 3,4 \\ Gerard E Francisco (iD ${ }^{5}$ \\ Virgílio Bento iD \\ Steven P Cohen (iD) ${ }^{6,7}$ \\ Fernando Dias Correia (iD) ${ }^{1,8}$ \\ 'SWORD Health Technologies, Inc, \\ Clinical Validation, Draper, UT, USA; \\ ${ }^{2}$ Institute for Cognitive Science, \\ University of Colorado Boulder, Boulder, \\ CO, USA; ${ }^{3}$ Rovisco Pais Medical and \\ Rehabilitation Centre, Tocha, Portugal; \\ ${ }^{4}$ Faculty of Medicine, Coimbra University, \\ Coimbra, Portugal; ${ }^{5}$ Department of \\ Physical Medicine and Rehabilitation, The \\ University of Texas Health Science \\ Center McGovern Medical School, and \\ TIRR Memorial Hermann, Houston, TX, \\ USA; ' ${ }^{6}$ Departments of Anesthesiology \& \\ Critical Care Medicine, Physical Medicine \\ and Rehabilitation, Neurology, and \\ Psychiatry and Behavioral Sciences, Johns \\ Hopkins School of Medicine, Baltimore, \\ MD, USA; ${ }^{7}$ Departments of \\ Anesthesiology and Physical Medicine and \\ Rehabilitation and Anesthesiology, \\ Uniformed Services University of the \\ Health Sciences, Bethesda, MD, USA; \\ ${ }^{8}$ Neurology Department, Centro \\ Hospitalar e Universitário do Porto, \\ Porto, Portugal
}

Correspondence: Fernando Dias Correia SWORD Health Technologies, Inc, Clinical Validation, 65 E Wadsworth Park Dr Ste 230, Draper, UT, 84020, USA

Tel + I 385-308-8034

$\mathrm{Fax}+1$ 80I-206-3433

Email fcorreia@swordhealth.com
Background: Chronic shoulder pain (SP) is responsible for significant morbidity, decreased quality of life and impaired work ability, resulting in high socioeconomic burden. Successful SP management is dependent on adherence and compliance with effective evidence-based interventions. Digital solutions may improve accessibility to such treatments, increasing convenience, while reducing healthcare-related costs.

Purpose: Present the results of a fully remote digital care program (DCP) for chronic SP. Patients and Methods: Interventional, single-arm, cohort study of individuals with chronic SP applying for a digital care program. Primary outcome was the mean change between baseline and 12 weeks on the Quick Disabilities of the Arm, Shoulder and Hand (QuickDASH) questionnaire. Secondary outcomes were change in pain (NPRS), analgesic consumption, intention to undergo surgery, anxiety (GAD-7), depression (PHQ-9), fearavoidance beliefs (FABQ-PA), work productivity (WPAI) and engagement.

Results: From 296 patients at program start, 234 (79.1\%) completed the intervention. Changes in QuickDASH between baseline and end-of-program were both statistically $(p<0.001)$ and clinically significant, with a mean reduction of $51.6 \%$ (mean -13.45 points, $95 \%$ CI: 11.99 ; 14.92). Marked reductions were also observed in all secondary outcomes: $54.8 \%$ in NPRS, $44.1 \%$ ceased analgesics consumption, $55.5 \%$ in surgery intent, $37.7 \%$ in FABQ-PA, $50.3 \%$ in anxiety, $63.6 \%$ in depression and $66.5 \%$ in WPAI overall. Higher engagement was associated with higher improvements in disability. Mean patient satisfaction score was 8.7/10.0 (SD 1.6).

Conclusion: This is the first real-world cohort study reporting the results of a multimodal remote digital approach for chronic SP rehabilitation. High completion and engagement rates were observed, which were associated with clinically significant improvement in all healthrelated outcomes, as well as marked productivity recovery. These promising results support the potential of digital modalities to address the global burden of chronic musculoskeletal pain.

Keywords: chronic pain, physical therapy, telerehabilitation, digital therapeutic, eHealth

\section{Plain Summary}

Chronic shoulder pain is one of the most frequent complaints in primary care, and has a huge impact on health, work capability and quality of life.

Current recommendations advise conservative management, namely exercise, as the first-line approach. However, there are several barriers to care access, namely local availability, time and travel constraints. Telerehabilitation may solve these challenges by delivering interventions remotely, improving access and engaging patients in their own rehabilitation while reducing costs. Telerehabilitation has shown similar results to 


\section{Graphical Abstract}

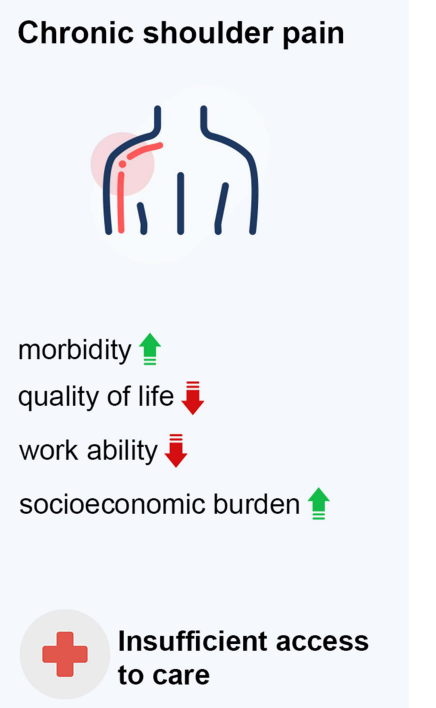

Single-arm prospective longitudinal cohort study

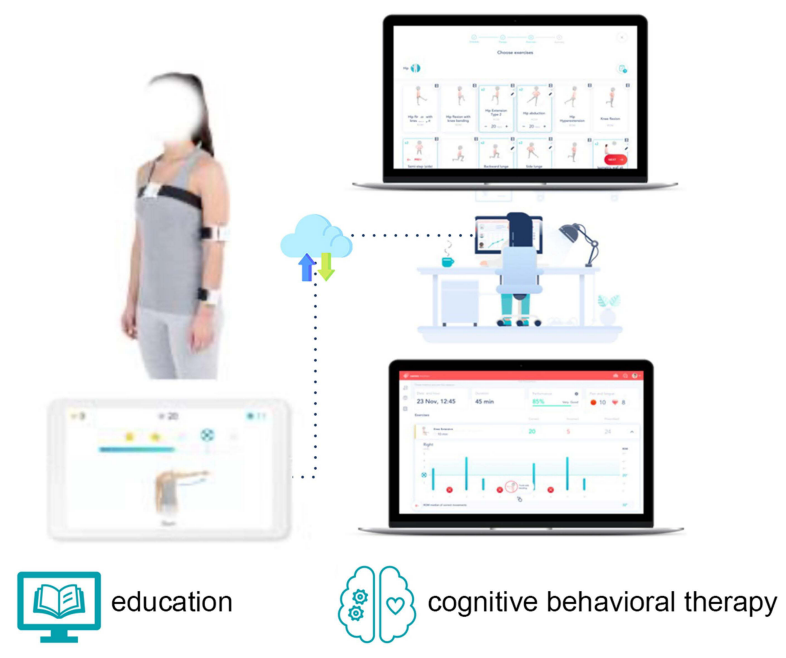

Asynchronous remote telerehabilitation
Observed outcomes

Disability

$51.6 \%$

Pain $\overline{\bar{N}}$

$54.8 \%$

Ceased medication

$44.1 \%$

Surgery intent $\overline{\bar{\nabla}}$

$55.5 \%$

Anxiety $\overline{\bar{V}}$

$50.3 \%$

Depression $\overline{\bar{\nabla}}$

$63.6 \%$

Productivity losses $\overline{\overline{7}}$ $66.5 \%$

Patient satisfaction $8.7 / 10.0$ in-person care, but it is still not well explored in the management of chronic shoulder pain, with interventions missing communication and/or biofeedback features.

In this study, we assessed the progress of a large group of patients going through a home-based telerehabilitation program managed remotely and asynchronously by an assigned physical therapist. This program integrates exercise, education on chronic shoulder pain, and also offers tools to manage anxiety and pain coping, both through recorded audio sessions including meditation and through self-paced tasks. Exercises are guided through a tablet and motion trackers that digitize movements and provide real-time feedback during each exercise through audio and video.

We report meaningful reductions in disability (51.6\%), pain $(54.8 \%)$, anxiety and depression $(50.3-63.6 \%)$, productivity $(66.5 \%)$ and willingness to undergo surgery $(55.5 \%)$, which were associated with high engagement and satisfaction levels.

This study supports the utility of telerehabilitation namely of digital care programs - in the management of shoulder conditions, showing results which are comparable to those reported for in-person care.

\section{Introduction}

Shoulder pain (SP) has a lifetime prevalence of nearly $70 \%{ }^{1}$ and is therefore one of the most common musculoskeletal (MSK) complaints in primary care. ${ }^{2}$ Importantly, after a first episode of shoulder pain, about $50 \%$ of cases become chronic, ${ }^{1}$ making SP one of the most prevalent causes of chronic MSK pain. ${ }^{3}$

Chronic SP is a significant cause of morbidity with tremendous impact on quality of life, psychological distress, and work ability. ${ }^{4-6}$ Indeed, a study performed in over 1 million Finnish employees found shoulder disorders to be the second most common cause of absenteeism among musculoskeletal conditions. ${ }^{7}$ Individuals with disabling shoulder disorders are expected to lose between 1.8 and 8.1 years of working life. ${ }^{8}$ Substantial social burden arises therefore not only from utilization of healthcare resources but also from productivity decline, ${ }^{6}$ with sick leaves contributing massively for SP costs. ${ }^{9}$

Guidelines advise exercise-based treatments as first-line approach for most SP conditions, ${ }^{10-13}$ with treatment directed not at the specific pathology but at movement dysfunction and potential underlying mechanisms. ${ }^{14,23,101}$ Previous research has shown comparable long-term outcomes between surgery and conservative treatment for most SP conditions $^{15-20}$ with recent guidelines ${ }^{21,100}$ adopting a critical view of surgical interventions versus conservative treatment. Recent research also recommends that key psychological factors (depression, anxiety, and fear-avoidance beliefs) be addressed as part of the intervention..$^{11,22,23}$ 
Successful SP management is dependent on the compliance with effective and evidence-based exercise treatments. ${ }^{24-28}$ Access to these is frequently challenged by local availability, time and travel constraints and high costs. $^{29,30}$ Telerehabilitation interventions have shown potential to ease these challenges, ${ }^{31-34}$ enabling equitable accessibility, higher adherence and improved quality, ${ }^{32,35,36}$ while reducing costs. ${ }^{37,38}$ Indeed, telerehabilitation has shown comparable results to conventional rehabilitation, with high satisfaction rates. ${ }^{39-42}$

However, SP telerehabilitation is still poorly explored, with scant literature on the subject. ${ }^{43-47}$ Most papers report the feasibility of different hardware/platforms ${ }^{48-53}$ or are pilot trials assessing pertinence of telerehabilitation strategies to deliver exercise programs, and only a few include built-in feedback/communication between patients and providers. ${ }^{44,50,54,55}$

We have developed a digital care program (DCP) which follows a multimodal biopsychosocial approach, integrating exercise, education, and cognitive behavioral therapy (CBT). This DCP is delivered through a platform combining a medical device that guides patients through the program and provides real-time biofeedback during exercises, with an assigned physical therapist (PT) asynchronously monitoring and tailoring the program.

This DCP was previously validated (feasibility and effectiveness $)^{39,40}$ in the rehabilitation of MSK conditions, including after shoulder tendon repair surgery, where it demonstrated comparable results to conventional therapy in the short term, and the ability to maximize clinical outcomes in the long term. ${ }^{42}$

In this study, we aim to describe the feasibility of this DCP on a real-world cohort of patients with chronic shoulder pain and the observed change in clinical outcomes (primarily disability, and in other health-related and productivity domains), engagement, and satisfaction. We hypothesize that the observed outcomes would be at least similar to those of other conventional rehabilitation programs reported in the literature.

\section{Methods}

\section{Study Design}

An interventional, single-arm, decentralized study, aiming to evaluate patients who underwent an entirely remote DCP for chronic SP, was performed between June 19th 2020 and August 2nd 2021. The study was conducted in accordance with the Declaration of Helsinki. Registration in ClinicalTrials.gov (NCT04092946, September 17th, 2019), and Approval from New England IRB (protocol number 120190313, June 18th, 2020) was prospectively obtained. All participants gave their informed consent prior to participation.

\section{Participants}

Adults ( $>18$ years old) suffering from chronic SP, covered by the health plans of 18 participating employers from 43 states in the United States of America (USA) were invited to apply to SWORD Health's digital MSK care program through a dedicated website. These programs were advertised to individuals using a variety of platforms, including home e-mail, mail, leaflets and posters.

Eligibility demanded for persistent or recurrent SP lasting longer than 12 weeks. Exclusion criteria included: a) fracture or significant trauma in the area of pain in the previous 3 months; b) shoulder surgery less than 3 months prior to enrollment; c) unexpected and rapidly progressive loss of strength or numbness in the upper limbs in the last 2 weeks; d) undergoing treatment for cancer; e) other health condition (eg, cardiac, respiratory) incompatible with at least 20 minutes of light to moderate exercise. Eligibility was confirmed during an initial video call by a physical therapist, which required the presence of chronic SP and the exclusion of patients with clinical redflags.

To control for selection bias, all those who enrolled in the DCP until 3rd May 2021 were considered.

\section{Intervention}

The DCP consisted of therapeutic exercise and education including CBT-related topics. Upon enrollment, all individuals were assigned a PT who developed a program tailored to each patient's needs. Patients performed exercise sessions independently using a class II medical device consisting of a tablet with a pre-installed app (with instructional videos) and wearable motion-tracking sensors. Motion trackers were placed using straps on the chest, upper arm, and wrist to digitize motion and provide realtime biofeedback during exercise. PTs remotely monitored participants' performance and made adjustments when necessary, through a web-based portal. Bi-directional communication was performed through a secure chat within a dedicated smartphone app (SWORD health app) or call. At least one touchpoint per week and one video call every 4 weeks were ensured. 
Participants were asked to perform at least 3 exercise sessions per week, with an expected program duration of 12 weeks, although early discharge was possible depending on PT assessment. Participants that, at any point, did not engage in any exercise session for 28 consecutive days were considered dropouts. Non-completers refer to participants that were excluded or dropped out after starting the program.

The educational component was delivered through the app, including educational articles (covering a broad range of chronic SP-related topics) and interactive modules based on CBT. Both components were developed under current clinical guidelines and research. The CBT program was created by a multidisciplinary team including psychiatrists and psychologists, based on third-generation CBT techniques - mindfulness, acceptance and commitment therapy and empathy-focused therapy. The CBT program was specifically designed to address fear-avoidance, pain reconceptualization, active coping skills, as well as anxiety and depression associated with musculoskeletal pain. The program consisted of self-guided interactive modules delivered through the smartphone app.

\section{Outcomes}

Outcomes were assessed through the mean change between baseline and end of program. Assessments were collected at baseline, 4, 8 and 12 weeks.

Primary outcome was patient-reported function, evaluated through Quick Disabilities of the Arm, Shoulder and Hand questionnaire (QuickDASH). QuickDASH consists of 11 items addressing disability and symptom severity, with scores ranging from 0 to $100 \%$, being higher scores related to worse functioning. ${ }^{56}$ An improvement of $30 \%$ was chosen as the minimal clinically important difference (MCID) ${ }^{57}$ Secondary outcome measures included:

- Numerical Pain Rating Scale (NPRS) through the question "Please rate your pain over the last 7 days: 0 (no pain at all) to 10 (worst pain imaginable)".

- Analgesic usage, assessed through the question: "Are you currently taking any pain medication?".

- Surgery intent, addressed through the question "How likely are you to have surgery to address your condition in the next 12 months?" (range 0 - not at all likely; 100 - extremely likely).

- Generalized Anxiety Disorder (GAD-7) 7-item scale (range $0-21)^{58}$ to assess anxiety, and Patient Health (PHQ-9) 9-item questionnaire (range 0-27) to assess depression. ${ }^{59,60} \mathrm{~A}$ threshold equal or greater than 5 was used to identify at least mild anxiety or depression.

- Fear-Avoidance Beliefs Questionnaire for physical activity (FABQ-PA), including a total of 4 items, each with a 7 option Likert scale (0-24). ${ }^{61,62}$

- Work Productivity and Activity Impairment questionnaire (WPAI) for general health, evaluated in employed participants, and assessing overall work impairment (overall), presenteeism (work), absenteeism (time) and activities impairment. ${ }^{63}$

- Engagement, assessed through users engaging with the program per week, number of completed sessions per week; total exercise time (minutes); educational articles read; and satisfaction (points), through the question: "On a scale from 0 to 10 , how likely is it that you would recommend this intervention to a friend or neighbor?".

\section{Safety and Adverse Events}

Patients were instructed to report any adverse events when they occurred to their PT. Additionally, pain and fatigue scores (graduated from 0 to 10) were collected at the end of each session and monitored remotely by the PT.

\section{Statistical Analysis}

Descriptive statistics were applied to characterize the study population and usability metrics. To assess differences in baseline characteristics between: a) completers and non-completers; b) early completers vs completers, c) highly engaged vs other participants, an independent samples $t$-test or one-way ANOVA with Bonferroni posthoc was used for quantitative variables and Chi-squared test for qualitative variables.

Latent growth curve analysis (LGCA) was used to model the trajectories of all outcome variables over time. Full information maximum likelihood estimation was used to deal with missing data. ${ }^{64-67}$ Trajectories were represented through intercept, slope and curvature for each variable. The intercept represents initial status at baseline and slope the estimated linear change in the outcome per week over time, while curvature indicates possible leveling of the effect. Parameters were adjusted for covariates (age, sex, body mass index (BMI)) and fitted as random effects allowing each to vary between individuals (see structural equation and path diagram for the LGCA used in Supplementary Figure 1). All models were estimated with a robust sandwich estimator for standard errors. This 
analysis was performed both for unfiltered and filtered cases at baseline considering the thresholds: $>0$ for surgery intent and WPAI; $\geq 5$ points for GAD-7 and PHQ-9. The LGCA was also applied to assess differences between early completers and completers. Additionally, a conditional analysis was performed to assess the influence of the age, sex, BMI, GAD-7, PHQ-9 as covariates. Both an intent-to-treat (ITT) and per protocol analysis were explored. Estimation of model fit was assessed through chi-squared test, root mean square error of approximation (RMSEA), confirmatory fit index (CFI), and standardized root mean square residual (SRMR). ${ }^{68,69}$

Logistic regression analysis was performed to identify the association of baseline clinical outcomes and engagement with responder status for QuickDASH.

Bivariate correlations (Pearson $r$ ) were used to investigate associations between outcomes. Significance levels were set at $\mathrm{p}<0.05$ in all analyses. LGCA was coded using $\mathrm{R}$ (version 1.4.1717) and all other analyses were performed using SPSS (version 17.0, SPSS Inc, Chicago, Illinois, USA).

\section{Results}

A total of 336 participants were screened for eligibility. From these, 296 (88.1\%) started the program, 0.9\% (3/ $336)$ declined consent, $0.3 \%(1 / 336)$ applied to the program but missed the enrollment video call, and $11.9 \%$ (27 $+13 / 336$ ) were excluded. Study flow diagram is presented in Figure 1. Program completion rate was 79.1\% (234/ $296)$, with $66.8 \%(175 / 262)$ complete data at 4 weeks, $68.3 \%(168 / 246)$ at 8 weeks and $63.9 \%(138 / 216)$ at 12 weeks.

\section{Baseline Characteristics}

Participant baseline characteristics $(\mathrm{N}=296)$ are presented in Table 1. No significant differences in demographic or clinical characteristics were observed between completers $(\mathrm{N}=234)$ and non-completers $(\mathrm{N}=62)$, except for baseline age, with completers being older than non-completers (51.7, SD 0.7 vs 47.7 SD, 1.7; p = 0.034) (Supplementary Table S1).

\section{Clinical Outcomes}

Latent Growth Curve (LGC) was applied to perform both an ITT $(\mathrm{N}=296)$ and a per-protocol $(\mathrm{N}=234)$ analysis. Per protocol results (Supplementary Table S2) were similar to ITT, and therefore this last more inclusive approach will be discussed.
Results of the ITT unconditional model (both for filtered and unfiltered data) are presented in Supplementary Table S3 and summarized in Table 2. Figure 2 depicts both mean trajectory and individual trajectories for each variable. Model fit calculations are presented in Supplementary Table S4. Results of the conditional model are detailed in Supplementary Table S5.

\section{Early vs Non-Early Completers}

Early completers had lower baseline scores in QuickDASH, GAD-7, PHQ-9, FABQ-PA, and WPAI activity, translating lower disability (Supplementary Table S6). They showed similar recovery trajectories in all outcomes, except for depression (PHQ-9), where they presented lower change but mean baseline was already very low in this group.

\section{QuickDASH}

Older participants, females, participants with higher BMI or with higher scores of PHQ-9 (depression) showed higher baseline scores $(\mathrm{p}<0.001$ or $\mathrm{p}<0.05$, Supplementary Table S5).

The mean overall change of QuickDASH was 13.45 points (95\% CI: $11.99 ; 14.92$, Table 2$)$, at a rate of 1.61 points per week (SD 1.40), representing a 51.6\% recovery from baseline ( $\mathrm{p}<0.001$, Supplementary Table S3 and Figure 2). Importantly, considering the MCID of $30 \%$ for QuickDASH, ${ }^{57}$ results show an odds ratio (OR) of 3.06, corresponding to $75.4 \%$ probability of a participant being a responder ( $\mathrm{p}<0.001$, Supplementary Table S7A).

Higher recovery was observed in participants with higher scores at baseline $(\mathrm{r}(134)=0.531, \mathrm{p}<0.001)$. Females had a faster pace of recovery $(-1.33$ per week, $\mathrm{p}=0.001)$ (Supplementary Table S5). No other covariates affected recovery trajectories, as well as OR for responders (Supplementary Table S7B).

\section{Pain}

The mean overall change in pain was 2.50 points $(95 \% \mathrm{CI}$ : $2.22 ; 2.77$ ), corresponding to a $54.8 \%$ improvement from baseline (Table 2), decreasing over time at an average of 0.41 points per week (SD 0.27; p $<0.001$, Figure 2 and Supplementary Table S3). Pain reduction was correlated with QuickDASH recovery $(\mathrm{r}(197)=0.390, \mathrm{p}<0.001)$. Higher pain recovery was observed in participants with higher pain and QuickDASH scores at baseline ( $\mathrm{r}(139)$ $=0.617, \mathrm{p}<0.001$ and $\mathrm{r}(139)=0.171, \mathrm{p}=0.044$, respectively). Recovery trend was not influenced by any covariates. 


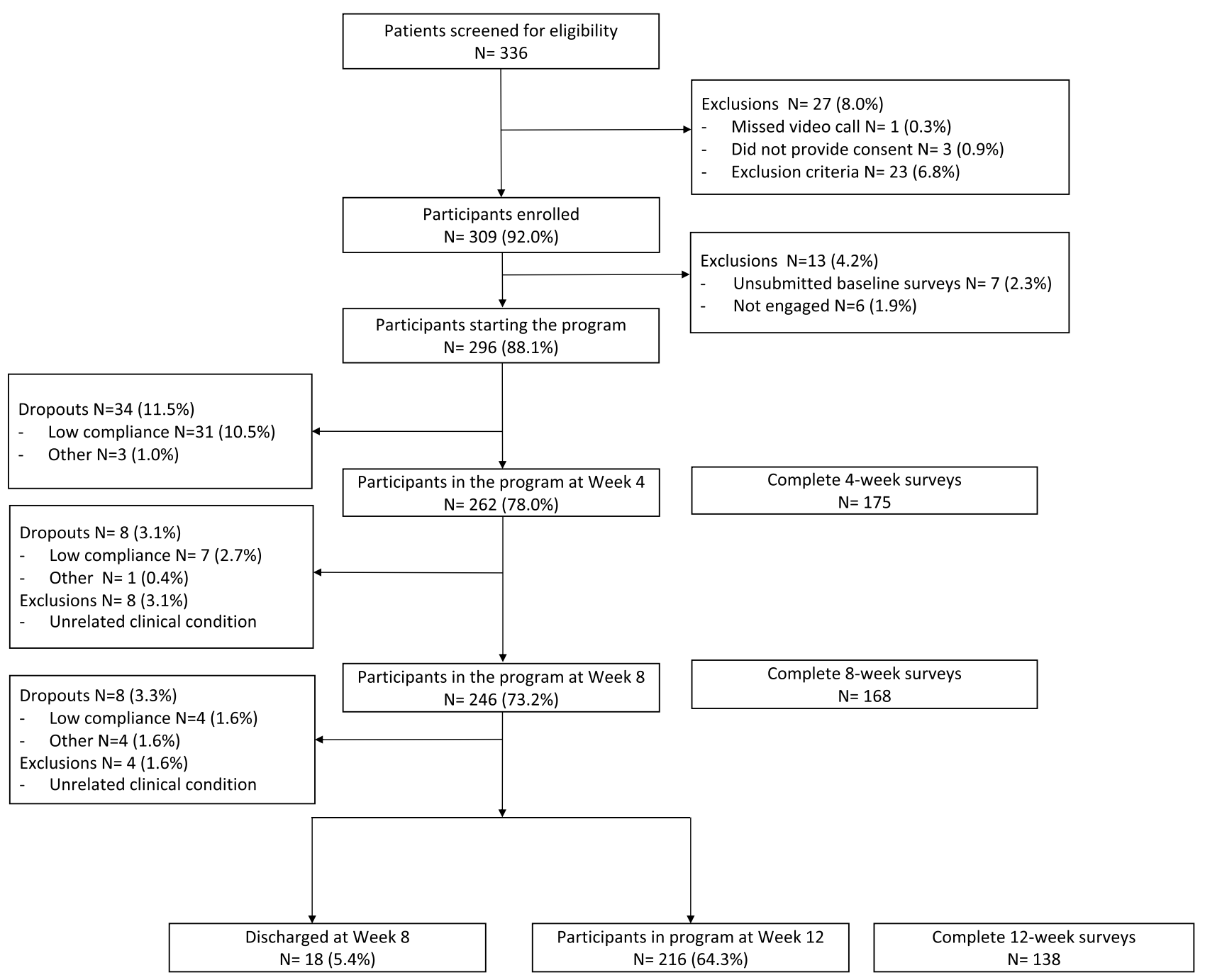

Figure I Study flow diagram.

\section{Medication Usage}

About 36.5\% (108/296) patients reported analgesics consumption at baseline. For participants where end of program data was available, $44.1 \%$ (26/59) of those taking medication at baseline had stopped consuming analgesics.

\section{Surgery Intent}

Surgery intent decreased at an average of 2.72 points (SD 2.45) per week, resulting in $55.5 \%$ reduction (13.53 mean change, $95 \%$ CI: $8.84 ; 18.23$ ) by end of program (Figure 2 and Table 2). A positive correlation was observed with the QuickDASH $(\mathrm{r}(197)=0.232, \mathrm{p}=0.001)$. Covariates had no impact on surgery intent.

\section{Mental Health and Fear-Avoidance Beliefs}

Participants that screened positive for at least mild anxiety at baseline (GAD-7 $\geq 5$ ) presented a significant reduction of 0.91 points (SD 0.29$)$ per week $(p<0.001)$, corresponding to an overall change of $50.3 \%$ (4.18 points, $95 \% \mathrm{CI}$ : $3.00 ; 5.36$ ) (Table 2). A similar rate was observed for participants with at least mild depression (PHQ-9 $\geq 5$ ), decreasing 0.88 points (SD 0.64) per week ( $<0.001$ ), corresponding to a reduction of $63.6 \%$ (4.92 points, $95 \%$ CI: 3.86 ; 5.99) (Table 2) at the end of the DCP. Anxiety and depression scores at baseline were positively and bidirectionally correlated $(p<0.001)$. Females presented lower depression levels at baseline compared to males (baseline $-1.52, \mathrm{p}=0.009$ ) (Supplementary Table S5). No other covariates had impact on anxiety and depression. 
Table I Baseline Characteristics of Study Participants ( $N=296)$

\begin{tabular}{|l|r|}
\hline Characteristic & Estimate \\
\hline Age (years), mean (SD) & $50.9(1 \mathrm{I} .6)$ \\
Age categories (years), N (\%): & \\
- <25 & $2(0.7)$ \\
- $25-40$ & $55(18.6)$ \\
- 40-60 & $169(57.1)$ \\
- > 60 & $70(23.6)$ \\
Sex (females), N (\%) & $156(52.7)$ \\
BMI, mean (SD) & $28.7(6.6)$ \\
BMI categories, N (\%): & \\
- Underweight (<18.5) & $3(1.0)$ \\
- Normal (18.5-25) & $86(29.1)$ \\
- Overweight (25-30) & $105(35.5)$ \\
- Obese (30-40) & $85(28.7)$ \\
- Obese grade III (>40) & $16(5.4)$ \\
Employment status, N (\%): & \\
- Employed (part-time or full-time) & $275(92.9)$ \\
- Unemployed (not working or seeking for & $21(7.1)$ \\
Opportunities) & \\
Occupation type, N (\%): & \\
- White collar & $184(62.2)$ \\
- Blue collar & $74(25.0)$ \\
- Not applicable (eg retired, unemployed) & $38(12.8)$ \\
Exercise level (days per week), N (\%): & \\
- None & $40(18.9)$ \\
- I-2 days & $46(21.7)$ \\
- 3-4 days $>5$ days & $49(23.3)$ \\
\hline
\end{tabular}

Abbreviation: BMI, body mass index.
Moderate fear-avoidance beliefs were observed at baseline (11.70, SD 4.41) and FABQ-PA scores decreased over time, at a pace of 0.32 points $(\mathrm{SD} 0.71)$ per week $(\mathrm{p}=$ $0.002)$, with a reduction of $37.7 \%$ by program end $(4.41$ mean change, 95\% CI: 3.55; 5.27), which was correlated to QuickDASH recovery $(\mathrm{r}(197)=0.273, \mathrm{p}<0.001)$ and to activity impairment $(\mathrm{r}(202)=0.231, \mathrm{p}<0.001)$. Older participants recovered faster $(-0.03$ per week, $\mathrm{p}=0.008)$ (Supplementary Table S5).

\section{Work Productivity}

Work productivity improved significantly across subscales analyzed - overall productivity, presenteeism and activity impairment (Table 2 and Figure 2). Very few participants reported absenteeism at baseline (27/251), and therefore were not submitted to LGC modeling, although paired samples $t$-test showed significant reduction from 17.28 (SD 11.85) to 2.43 (SD 6.86) at program end ( $\mathrm{p}<$ $0.001)$. Participants had similar recovery for both overall productivity and presenteeism $(\sim 67 \%)$ (Table 2$)$ with recovery pace of 2.71 and 2.45 points per week (both $\mathrm{p}<0.001)$, respectively.

A recovery of $56.3 \%$ (mean change 16.33 , 95\% CI: $12.80 ; 19.86)$ was also observed for activity impairment (Table 2). These results were correlated with QuickDASH improvement (overall: $\mathrm{r}(156)=0.402$; presenteeism: $\mathrm{r}$

Table 2 Changes in Clinical Outcomes Between Baseline and 12 Weeks: Unconditional Intent-to-Treat Approach

\begin{tabular}{|c|c|c|c|c|c|}
\hline Outcome, Mean $(95 \% \mathrm{Cl})$ & $\mathbf{N}$ & Baseline & End-of-Program & Mean Change & \% Change \\
\hline QuickDASH & 296 & $26.07(24.59 ; 27.55)$ & $12.62(11.08 ; 14.15)$ & 13.45 (11.99; 14.92) & $51.6 \%$ \\
\hline Pain Level & 296 & $4.56(4.35 ; 4.77)$ & $2.06(1.81 ; 2.32)$ & $2.50(2.22 ; 2.77)$ & $54.8 \%$ \\
\hline Surgery Intent $>0$ & 161 & $24.38(20.78 ; 27.98)$ & 10.85 (6.27; I5.42) & | 3.53 (8.84; 18.23) & $55.5 \%$ \\
\hline Surgery Intent & 296 & I3.27 (I0.87; 15.68) & $6.16(3.60 ; 8.73)$ & $7.11(4.36 ; 9.86)$ & $53.5 \%$ \\
\hline GAD-7 $\geq 5$ & 87 & $8.30(7.56 ; 9.05)$ & $4.12(3.03 ; 5.22)$ & $4.18(3.00 ; 5.36)$ & $50.3 \%$ \\
\hline GAD-7 & 296 & $3.34(2.89 ; 3.79)$ & I.87 (I.39; 2.35) & $1.47(0.95 ; 1.99)$ & $43.9 \%$ \\
\hline PHQ- $9 \geq 5$ & 79 & 7.74 (7.11; 8.38) & $2.82(I .93 ; 3.7 I)$ & $4.92(3.86 ; 5.99)$ & $63.6 \%$ \\
\hline PHQ-9 & 296 & $3.13(2.75 ; 3.5 \mathrm{I})$ & $1.47(1.12 ; 1.82)$ & $1.66(1.21 ; 2.11)$ & $53.0 \%$ \\
\hline FABQ-PA & 296 & $11.70($ (II.07; 12.32) & $7.29(6.48 ; 8.10)$ & $4.4 \mathrm{I}(3.55 ; 5.27)$ & $37.7 \%$ \\
\hline WPAI Overall >0 & 141 & $25.27(22.18 ; 28.35)$ & $8.46(4.6|;| 2.3 \mid)$ & $16.81(12.30 ; 21.31)$ & $66.5 \%$ \\
\hline WPAI Overall & 251 & I3.97 (II.68; 16.27) & $5.74(3.47 ; 8.01)$ & $8.24(5.37 ; 11.11)$ & $58.9 \%$ \\
\hline WPAI Work >0 & 136 & $23.46(20.62 ; 26.30)$ & 7.53 (3.87; II.18) & I5.93 (II.5I; 20.36) & $67.9 \%$ \\
\hline WPAI Work & 251 & $12.44(10.34 ; 14.54)$ & $5.58(3.32 ; 7.85)$ & $6.86(3.99 ; 9.72)$ & $55.1 \%$ \\
\hline WPAI Activity $>0$ & 236 & $29.01(26.59 ; 31.44)$ & $12.68(9.45 ; 15.91)$ & $16.33(12.80 ; 19.86)$ & $56.3 \%$ \\
\hline WPAI Activity & 296 & $23.03(20.69 ; 25.37)$ & $10.49(7.87 ; 13.10)$ & $12.54(9.65 ; 15.43)$ & $54.5 \%$ \\
\hline
\end{tabular}

Abbreviations: QuickDASH, Quick Disabilities of the Arm, Shoulder and Hand questionnaire; GAD-7, Generalized Anxiety Disorder 7-item scale; PHQ-9, Patient Health 9-item questionnaire; FABQ-PA, Fear-Avoidance Beliefs Questionnaire for physical activity; WPAI, Work Productivity and Activity Impairment questionnaire. 


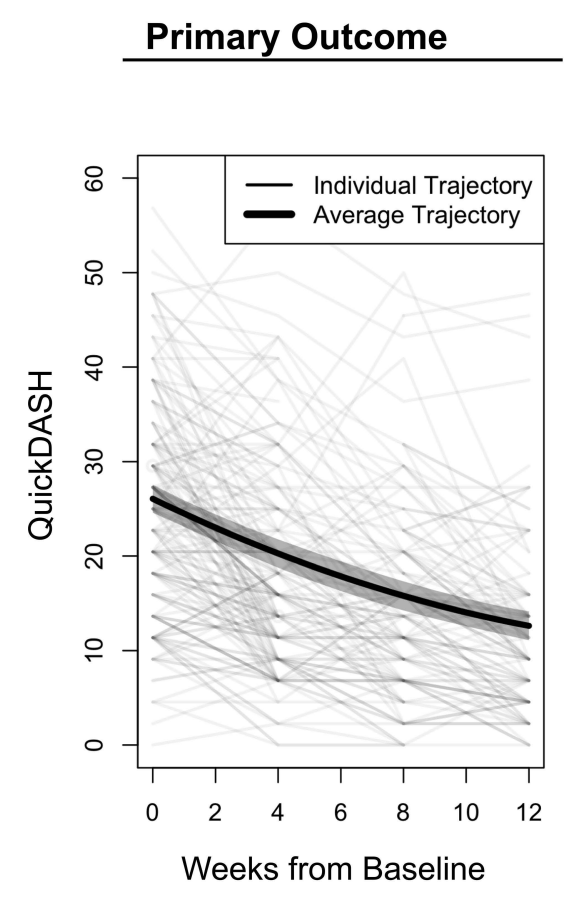

Secondary Outcomes
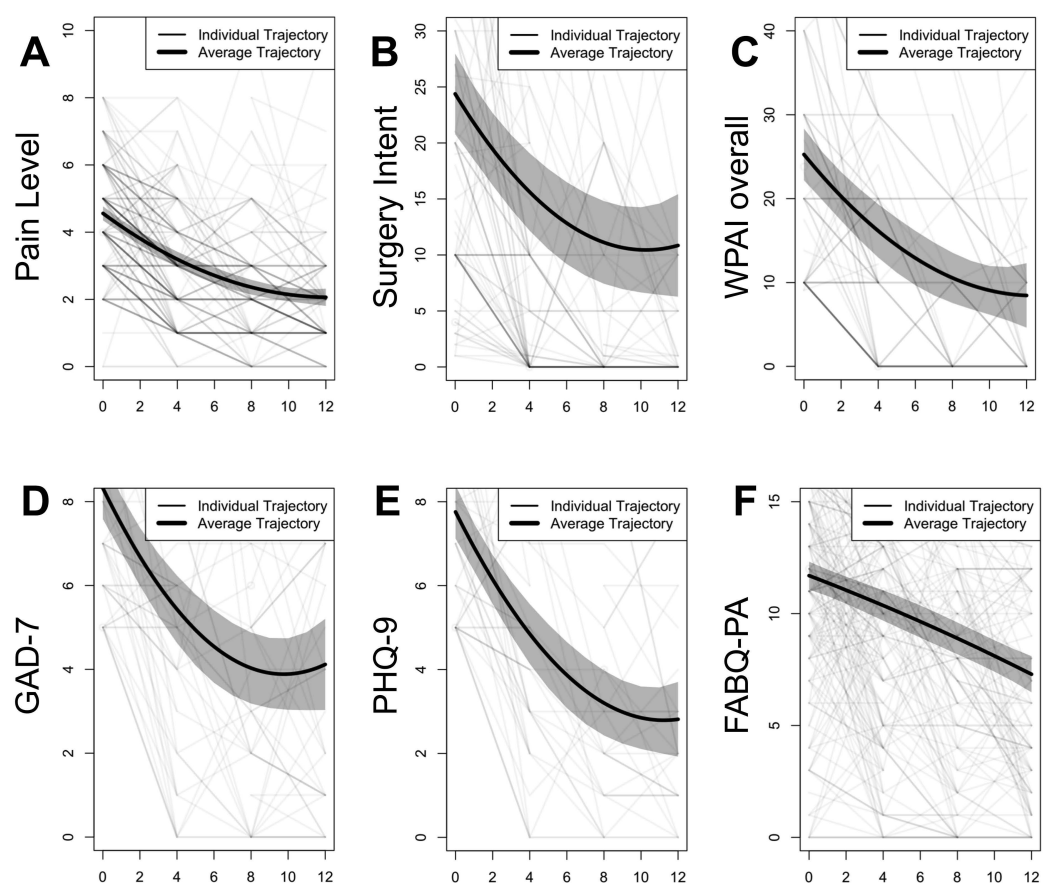

Figure 2 Longitudinal changes across time for all filtered variables. Primary outcome: QuickDASH; Secondary outcomes: (A) Pain level; (B) Surgery intent; (C) WPAI overall; (D) GAD-7; (E) PHQ-9; (F) FABQ-PA. Cases filtered according to the following baseline thresholds - surgery intent scores > 0 points; GAD-7 scores $\geq 5$ points; PHQ-9 scores $\geq 5$ points; WPAI overall $>0$ points.

$(156)=0.360$; activity impairment: $\mathrm{r}(197)=0.432$; all $\mathrm{p}<$ $0.001)$.

Regarding the effect of covariates, individuals with higher levels of depression had higher overall productivity $(\mathrm{p}=0.002)$, presenteeism $(\mathrm{p}=0.028)$ and activity impairment at baseline $(p=0.007)$, but with no influence on recovery pace.

\section{Engagement and Usability-Related Outcomes}

Participants performed on average 2.7 sessions per week (SD 1.5), and 40.5\% (120/296) completed at least 3 sessions per week (highly engaged participants - HEP). Mean exercise dosage was 763.7 minutes (SD 546.9). Participants read on average 10.4 educational content pieces (SD 10.2). Importantly, HEP achieved significant greater improvements in QuickDASH (-14.22, SD 9.01 vs -10.58 , SD 11.72) $(\mathrm{p}=0.016)$ and pain $(-2.92$, SD $1.57 \mathrm{vs}$ -1.98 , SD 2.16) $(\mathrm{p}<0.001)$. This was evidenced by the positive correlation between the average number of sessions per week and reduction in pain $(\mathrm{r}(202)=0.203, \mathrm{p}=$ 0.004), QuickDASH $(\mathrm{r}(197)=0.145, \mathrm{p}=0.042)$, and response to treatment (OR 1.51, 95\% CI 1.08; 2.17; $\mathrm{p}=$ 0.021) (Supplementary Table S7B). Notably, HEP were older (48.0, SD 11.9 vs 55.1, SD 9.7) (p < 0.001), presented lower BMI (27.8, SD 6.2 vs 29.3, SD 6.8) $(\mathrm{p}=$ $0.045)$ and lower obesity rates $(22 / 120$ vs $63 / 176)(\mathrm{p}=$ 0.003 ), and reported higher satisfaction levels (9.0, SD 1.2 vs 8.5 , SD 1.5) ( $\mathrm{p}=0.001)$. No other significant differences were observed. Overall, high satisfaction rates were reported with $62.7 \%(180 / 287)$ participants classifying the program as "9" or "10" and an average of 8.7 (SD 1.6).

\section{Discussion}

\section{Main Findings}

The DCP herein explored was able to promote very high engagement and compliance, which was correlated with clinically meaningful improvements in both disability (mean change of $51.6 \%$ in QuickDASH) and pain (54.8\% reduction), and an OR of 3.06 for being a responder regarding the primary outcome. Surgery intent was significantly reduced $(55.5 \%)$ which is in line with recommendations favoring non-surgical management of chronic SP conditions. ${ }^{10-13}$ The pertinence of the multimodal approach was further reinforced by the improvements in anxiety, depression, and fear-avoidance beliefs $(50.3 \%, 63.6 \%$ and $37.7 \%$, respectively). The improvements in all the above resulted in a substantial impact on 
productivity, with an average reduction in productivity losses of $66.5 \%$, suggesting that a fully remote DCP may contribute to reducing both the direct and indirect burden of chronic shoulder conditions.

\section{Comparison with the Literature}

Most studies on telerehabilitation in this area are small pilot clinical trials testing its feasibility for chronic shoulder conditions, with either very small sample size $(\mathrm{N}<30),{ }^{44,50,55}$ mixed chronic and acute pain population $^{45}$ or much shorter interventions (4-6 weeks). ${ }^{51,70}$ Malliaras et $\mathrm{al}^{54}$ conducted a randomized controlled trial $(\mathrm{RCT})(\mathrm{N}=36)$ comparing (i) advice only, (ii) recommended care (internet-delivered exercise and education) or (iii) recommended care and telerehabilitation. The third group obtained the highest recoveries in Shoulder Pain and Disability Index (SPADI) (69.1\%) and VAS pain (52.9\%). These results are higher than those reported in the present study for disability, and comparable to those reported for pain, but might be the result of much higher baseline disability and very small group size $(\mathrm{N}=12)$. Considering studies published on the impact of conventional (face-to-face) care, the results obtained in this study for QuickDASH - 51.6\% improvement (13.45, 95\% CI: $11.99 ; 14.92)$ - were higher than those reported by some studies $(17.0-37.5 \%)^{18,71-74}$ and in line with the results reported by others $(51.2-61 \%)^{75,76}$ - the latter with higher exercise dosages. Regarding secondary outcomes, the pain reduction reported in this study $-54.8 \%$ $(2.50$ points $95 \%$ CI: $2.22 ; 2.77)$ - was higher than that reported for in-person rehabilitation studies (26.5$46.4 \%) .{ }^{18,71,72}$ The accompanying reduction in the percentage of participants taking analgesics reinforces not only the impact on pain, but also the effectiveness of nonpharmacological interventions in the management of shoulder conditions. ${ }^{77-79}$

We also noted a marked shift in the willingness to undergo surgery (55.5\% decrease), reinforcing the importance of conservative care as recommended by current guidelines. $^{10,80}$ This is especially important in the light of the report by Kuhn et $\mathrm{al}^{81}$ that if a patient does not elect surgery within the first 12-weeks of non-operative rehabilitation, they are unlikely to require surgery for up to two years.

Mental health is a crucial aspect on chronic SP conditions and has been associated with poorer prognosis. ${ }^{82-85}$ Evidence shows exercise-based therapies can improve mental health indicators ${ }^{25,86}$ and that exercise programs supervised by PTs (versus home exercise) can improve mental health outcomes to a greater extent. ${ }^{22}$ The multimodal DCP herein presented was able to promote greater improvements in anxiety than those reported in other studies $(50.3 \%$ vs $44 \%)$, as well as substantial improvements in depression and fear-avoidance (FABQ-PA), which in turn correlated with improvements in QuickDASH and activity impairment. These findings support the inclusion of a biopsychosocial component into the treatment plan, as also described in other studies. ${ }^{11,14,87,88}$

Chronic SP may have a very significant impact in both short- and long-term work disability. ${ }^{89}$ This DCP promoted significant reductions in work-related productivity losses, with both WPAI overall and presenteeism presenting a reduction of change of $\sim 67 \%$. The low reported absenteeism is consistent with literature demonstrating that many workers still go to work despite MSK pain. ${ }^{90,91}$

Regarding engagement, the importance of consistency and high compliance with an exercise-based program is well recognized in the management of shoulder conditions. ${ }^{24-28}$ However, adherence is often poor and typically worse for unsupervised home exercise programs. ${ }^{92}$ In this study, the completion rate of $79.1 \%$ is higher than most studies published

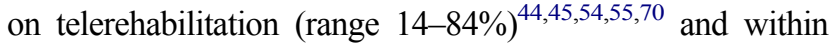
the range of those reported in conventional therapies RCTs (52-94\%). ${ }^{18,71-73,75,76}$ The high engagement observed in this study might be explained by the continuous PT monitoring, the direct communication channel and real-time biofeedback. All these factors were previously reported as key for patient compliance and engagement. ${ }^{22,45,54,55,93,94}$ Not surprisingly, high compliance was correlated with better recovery (in both disability and pain), reinforcing the need to formally address patient engagement in all programs.

\section{Engagement and Completion}

Regarding these topics, this study reported several interesting findings, the most striking aspect is completers being older than non-completers, and with a much greater proportion of patients above 60 years old. Also, highly engaged patients were older. These two facts challenge the notion of lower adoption of digital health by an older population. As expected, early completers corresponded to a less disabled population.

\section{Strengths and Limitations}

The strengths of the study include the large sample size and sample diversity, with participants from 43 different states within the USA. Unlike similar studies, this 
multimodal DCP addressed not only exercise but also education and mental health aspects, as supported by recent evidence. ${ }^{11,87,95}$ The innovative nature of this DCP also allowed for flexibility and convenience of treatment, while the real-time biofeedback, the biopsychosocial framework $^{29,96}$ and regular communication with the PT contributed to enhanced adherence and maximized clinical outcomes. $^{26,97}$ We utilized a comprehensive set of outcome measures, comprising several domains and using valid and reliable outcome measures, ${ }^{58,60,61,63,98}$ including for engagement, which was objectively measured rather than through self-reported outcomes which may create social desirability response bias. ${ }^{99}$ Additionally, preliminary responder analysis revealed promising results which will be addressed in a future RCT.

The absence of a control group is the most relevant study limitation as it would control for the natural course shoulder pain. However, given the real-world context in which this was performed, the most obvious comparator would be a "waiting list" control, which was not ethical considering the high accessibility this technology affords. Another limitation is the lack of long-term follow-up to assess full recovery and relapse rates.

\section{Recommendations for Future Studies}

The promising results presented here should be explored further in RCTs comparing the DCP with in-person PT or other digital programs, and ideally feature longer-term follow-up assessments.

\section{Conclusions}

This is the first study providing preliminary insights of the potential benefits of a structured and multimodal DCP in the management of chronic SP through a large real-world cohort. Very high adherence rates and patient satisfaction were observed, suggesting the feasibility of this approach in a real-world scenario. Substantial changes in disability, pain intensity, medication intake, surgery intent, mental health and productivity were achieved on par with those of RCTs involving conventional physical therapy. We believe digital modalities hold great promise in the delivery of accessible and effective rehabilitation, addressing the global burden of chronic pain.

\section{Abbreviations}

ANOVA, analysis of variance; BMI, body mass index; CBT, cognitive behavioral therapy; CFI, confirmatory fit index; CI, confidence interval; DCP, digital care program; FABQ-PA, Fear-Avoidance Beliefs Questionnaire for physical activity; GAD-7, Generalized Anxiety Disorder 7-item questionnaire; ITT, intent-totreat; LGCA, Latent growth curve analysis; MCID, minimal clinically important difference; MSK, Musculoskeletal; NPRS, Numerical Pain Rating Scale; OR, Odds ratio; PHQ-9, Patient Health 9-item questionnaire; PT, Physical therapist; QuickDASH, Quick Disabilities of the Arm, Shoulder and Hand; RCT, Randomized controlled trial; RMSEA, Root mean square error of approximation; SP, Shoulder pain; SPADI, Shoulder Pain and Disability Index; SRMR, Standardized root mean square residual; USA, United States of America; WPAI, Work Productivity and Activity Impairment questionnaire.

\section{Data Sharing Statement}

All data relevant to the study are included in the article or are available as Digital Content at Supplementary Material. Only de-identified individual participant data is provided. Further information, including the study protocol, can be found at ClinicalTrials.gov (NCT04092946).

\section{Ethics Approval and Informed Consent}

The study was approved by the New England IRB (protocol number 120190313) and prospectively registered in ClinicalTrials.gov, NCT04092946, 17/09/2019. This study was conducted in accordance with the approved guidelines. All patients were informed about the purpose and procedures of the study and provided informed consent.

\section{Acknowledgments}

The authors acknowledge the team of physical therapists responsible for the management of participants. The authors also acknowledge the contributions of João Tiago Silva and Quemuel Araújo in data validation (both employed at SWORD Health).

\section{Author Contributions}

All authors made a significant contribution to the work reported, whether that is in the conception, study design, execution, acquisition of data, analysis and interpretation, or in all these areas; took part in drafting, revising or critically reviewing the article; gave final approval of the version to be published; have agreed on the journal to 
which the article has been submitted; and agree to be accountable for all aspects of the work.

\section{Funding}

This research received no specific grant from any funding agency in the public, commercial or not-for-profit sectors.

\section{Disclosure}

Dora Janela, Fabíola Costa, Maria Molinos, Virgílio Bento and Fernando Dias Correia are employees at SWORD Health, the study sponsor. Robert Glen Moulder, Jorge Lains, Gerard E Francisco and Steven Paul Cohen are independent scientific consultants who were funded by SWORD Health in connection with the development and execution of this article. In addition Dr Steven Paul Cohen reports personal fees from Releviate, personal fees from Persica, grants from Scilex, outside the submitted work. The authors report no other conflicts of interest in this work.

\section{References}

1. Luime JJ, Koes BW, Hendriksen IJ, et al. Prevalence and incidence of shoulder pain in the general population; a systematic review. Scand J Rheumatol. 2004;33(2):73-81. doi:10.1080/03009740310004667

2. Jordan KP, Kadam Ut, Hayward R, Porcheret M, Young C, Croft P. Annual consultation prevalence of regional musculoskeletal problems in primary care: an observational study. BMC Musculoskelet Disord. 2010;11(1):144. doi:10.1186/1471-2474-11-144

3. United States Bone and Joint Initiative. The Burden of Musculoskeletal Diseases in the United States (BMUS) [homepage on the Internet]. Musculoskeletal Conditions. Available from: http:// www.boneandjointburden.org. Accessed May 14, 2021.

4. Imagama S, Ando K, Kobayashi K, et al. Shoulder pain has most impact on poor quality of life among various types of musculoskeletal pain in middle-aged and elderly people: yakumo study. Mod Rheumatol. 2020;30(3):568-572. doi:10.1080/ 14397595.2019.1623364

5. Martimo KP, Shiri R, Miranda H, Ketola R, Varonen H, ViikariJuntura E. Self-reported productivity loss among workers with upper extremity disorders. Scand J Work Environ Health. 2009;35 (4):301-308. doi:10.5271/sjweh.1333

6. Ackerman IN, Fotis K, Pearson L, et al. Impaired health-related quality of life, psychological distress, and productivity loss in younger people with persistent shoulder pain: a cross-sectional analysis Disabil Rehabil. 2021;23:1-10. doi:10.1080/09638288.2021.1887376

7. Pekkala J, Rahkonen O, Pietiläinen O, Lahelma E, Blomgren J. Sickness absence due to different musculoskeletal diagnoses by occupational class: a register-based study among 1.2 million Finnish employees. Occup Environ Med. 2018;75(4):296. doi:10.1136/ oemed-2017-104571

8. Sirén M, Viikari-Juntura E, Arokoski J, Solovieva S. Work participation and working life expectancy after a disabling shoulder lesion Occup Environ Med. 2019;76(6):363. doi:10.1136/oemed-2018105647

9. Virta L, Joranger P, Brox JI, Eriksson R. Costs of shoulder pain and resource use in primary health care: a cost-of-illness study in Sweden. BMC Musculoskelet Disord. 2012;13(1):17. doi:10.1186/1471-2474$13-17$
10. Hopman K, Lukersmith S, Krahe L, McColl A, Vine K. Clinical practice guidelines for the management of rotator cuff syndrome in the workplace. Australian Occupational Therapy Journal. 2013;60(6):378-386. doi:10.1111/1440-1630.12077

11. Kelley MJ, Shaffer MA, Kuhn JE, et al. Shoulder pain and mobility deficits: adhesive capsulitis. J Orthop Sports Phys Ther. 2013;43(5):A1-31. doi:10.2519/jospt.2013.0302

12. New York State Workers' Compensation Board. New York Shoulder Injury Medical Treatment Guidelines; 2014.

13. Doiron-Cadrin P, Lafrance S, Saulnier M, et al. Shoulder rotator cuff disorders: a systematic review of clinical practice guidelines and semantic analyses of recommendations. Arch Phys Med Rehabil. 2020;101(7):1233-1242. doi:10.1016/j. apmr.2019.12.017

14. Lewis J. Rotator cuff related shoulder pain: assessment, management and uncertainties. Man Ther. 2016;23:57-68. doi:10.1016/j. math.2016.03.009

15. Saltychev M, Äärimaa V, Virolainen P, Laimi K. Conservative treatment or surgery for shoulder impingement: systematic review and meta-analysis. Disabil Rehabil. 2015;37(1):1-8. doi:10.3109/ 09638288.2014.907364

16. Ranebo MC, Björnsson Hallgren HC, Holmgren T, Adolfsson LE. Surgery and physiotherapy were both successful in the treatment of small, acute, traumatic rotator cuff tears: a prospective randomized trial. J Shoulder Elbow Surg. 2020;29(3):459-470. doi:10.1016/j.jse.2019.10.013

17. Lähdeoja T, Karjalainen T, Jokihaara J, et al. Subacromial decompression surgery for adults with shoulder pain: a systematic review with meta-analysis. $\mathrm{Br} J$ Sports Med. 2020;54(11):665. doi:10.1136/bjsports-2018-100486

18. Rangan A, Brealey SD, Keding A, et al. Management of adults with primary frozen shoulder in secondary care (UK FROST): a multicentre, pragmatic, three-arm, superiority randomised clinical trial. Lancet. 2020;396(10256):977-989. doi:10.1016/s01406736(20)31965-6

19. Kukkonen J, Joukainen A, Lehtinen J, et al. Treatment of non-traumatic rotator cuff tears: a randomised controlled trial with one-year clinical results. Bone Joint J. 2014;96-b(1):75-81. doi:10.1302/0301-620x.96b1.32168

20. Kukkonen J, Joukainen A, Lehtinen J, et al. Treatment of nontraumatic rotator cuff tears: a randomized controlled trial with two years of clinical and imaging follow-up. J Bone Joint Surg Am. 2015;97(21):1729-1737. doi:10.2106/jbjs.N.01051

21. Vandvik PO, Lähdeoja T, Ardern C, et al. Subacromial decompression surgery for adults with shoulder pain: a clinical practice guideline. BMJ. 2019;364:1294. doi:10.1136/bmj.1294

22. Russell S, Jariwala A, Conlon R, Selfe J, Richards J, Walton M. A blinded, randomized, controlled trial assessing conservative management strategies for frozen shoulder. J Shoulder Elbow Surg. 2014;23(4):500-507. doi:10.1016/j.jse.2013.12.026

23. Lewis J, McCreesh K, Roy JS, Ginn K. Rotator cuff tendinopathy: navigating the diagnosis-management conundrum. J Orthop Sports Phys Ther. 2015;45(11):923-937. doi:10.2519/ jospt.2015.5941

24. Edwards P, Ebert J, Joss B, Bhabra G, Ackland T, Wang A. Exercise rehabilitation in the non-operative management of rotator cuff tears: a review of the literature. Int J Sports Phys Ther. 2016;11(2):279-301.

25. Hanratty CE, McVeigh JG, Kerr DP, et al. The effectiveness of physiotherapy exercises in subacromial impingement syndrome: a systematic review and meta-analysis. Semin Arthritis Rheum. 2012;42(3):297-316. doi:10.1016/j.semarthrit.2012.03.015

26. Jordan JL, Holden MA, Mason EE, Foster NE. Interventions to improve adherence to exercise for chronic musculoskeletal pain in adults. Cochrane Database Syst Rev. 2010;2010(1):CD005956. doi:10.1002/14651858.CD005956.pub2 
27. Pieters L, Lewis J, Kuppens K, et al. An update of systematic reviews examining the effectiveness of conservative physical therapy interventions for subacromial shoulder pain. J Orthop Sports Phys Ther. 2020;50(3):131-141. doi:10.2519/jospt.2020.8498

28. Hall K, Grinstead A, Lewis JS, Mercer C, Moore A, Ridehalgh C. Rotator cuff related shoulder pain. Describing home exercise adherence and the use of behavior change interventions to promote home exercise adherence: a systematic review of randomized controlled trials. Phys Therapy Rev. 2021;26(4):299-322. doi:10.1080/10833196.2021.1935106

29. Lin I, Wiles L, Waller R, et al. What does best practice care for musculoskeletal pain look like? Eleven consistent recommendations from high-quality clinical practice guidelines: systematic review. $\mathrm{Br}$ J Sports Med. 2020;54(2):79-86. doi:10.1136/bjsports-2018-099878

30. Carvalho E, Bettger JP, Goode AP. Insurance coverage, costs, and barriers to care for outpatient musculoskeletal therapy and rehabilitation services. $N \quad C$ Med J. 2017;78(5):312-314. doi:10.18043/ncm.78.5.312

31. Adamse C, Dekker-van Weering MG, van Etten-jamaludin FS, Stuiver MM. The effectiveness of exercise-based telemedicine on pain, physical activity and quality of life in the treatment of chronic pain: a systematic review. J Telemed Telecare. 2018;24 (8):511-526. doi:10.1177/1357633x17716576

32. Cottrell MA, Galea OA, O’Leary SP, Hill AJ, Russell TG. Realtime telerehabilitation for the treatment of musculoskeletal conditions is effective and comparable to standard practice: a systematic review and meta-analysis. Clin Rehabil. 2017;31 (5):625-638. doi:10.1177/0269215516645148

33. Lin HT, Li YI, Hu WP, Huang CC, Du YC, Scoping A. Review of the efficacy of virtual reality and exergaming on patients of musculoskeletal system disorder. J Clin Med. 2019;8:6. doi:10.3390/jcm8060791

34. Heapy AA, Higgins DM, Cervone D, et al. Review of technology-assisted self-management interventions for chronic pain: looking across treatment modalities. Clin J Pain. 2015;31 (6):470-492. doi:10.1097/ajp.0000000000000185

35. Tenforde AS, Hefner JE, Kodish-Wachs JE, Iaccarino MA, Paganoni S. Telehealth in physical medicine and rehabilitation: a narrative review. $P m$ r. 2017;9(5s):S51-s58. doi:10.1016/j. pmrj.2017.02.013

36. Turolla A, Rossettini G, Viceconti A, Palese A, Geri T. Musculoskeletal Physical Therapy During the COVID-19 Pandemic: is Telerehabilitation the Answer? Phys Ther. 2020;100(8):1260-1264. doi:10.1093/ptj/pzaa093

37. Fatoye F, Gebrye T, Fatoye $\mathrm{C}$, et al. The clinical and cost-effectiveness of telerehabilitation for people with nonspecific chronic low back pain: randomized controlled trial. JMIR Mhealth Uhealth. 2020;8(6):e15375. doi:10.2196/15375

38. Pastora-Bernal JM, Martín-Valero R, Barón-López FJ. Cost analysis of telerehabilitation after arthroscopic subacromial decompression. $J$ Telemed Telecare. 2017;24(8):553-559. doi:10.1177/1357633X17723367

39. Correia FD, Nogueira A, Magalhaes I, et al. Home-based rehabilitation with a novel digital biofeedback system versus conventional in-person rehabilitation after total knee replacement: a feasibility study. Sci Rep. 2018;8(1):11299. doi:10.1038/ s41598-018-29668-0

40. Dias Correia F, Nogueira A, Magalhaes I, et al. Digital Versus Conventional Rehabilitation After Total Hip Arthroplasty: a Single-Center, Parallel-Group Pilot Study. JMIR Rehabil Assist Technol. 2019;6(1):e14523. doi:10.2196/14523

41. Correia FD, Nogueira A, Magalhaes I, et al. Medium-Term Outcomes of Digital Versus Conventional Home-Based Rehabilitation After Total Knee Arthroplasty: prospective, Parallel-Group Feasibility Study. JMIR Rehabil Assist Technol. 2019;6(1):e13111. doi:10.2196/13111
42. Correia FD, Molinos M, Luís S, et al. Digitally assisted versus conventional home-based rehabilitation after Arthroscopic Rotator Cuff Repair: a randomized controlled trial. Am J Phys Med Rehabil. 2021. doi:10.1097/phm.0000000000001780

43. Pastora-Bernal JM, Martín-Valero R, Barón-López FJ, Moyano NG, Estebanez-Pérez MJ. Telerehabilitation after arthroscopic subacromial decompression is effective and not inferior to standard practice: preliminary results. $J$ Telemed Telecare. 2018;24(6):428-433. doi:10.1177/1357633×17706583

44. Chen YP, Lin CY, Tsai MJ, Chuang TY, Lee OK. Wearable motion sensor device to facilitate rehabilitation in patients with shoulder adhesive capsulitis: pilot study to assess feasibility. J Med Internet Res. 2020;22(7):e17032. doi:10.2196/17032

45. Choi Y, Nam J, Yang D, Jung W, Lee HR, Kim SH. Effect of smartphone application-supported self-rehabilitation for frozen shoulder: a prospective randomized control study. Clin Rehabil. 2019;33(4):653-660. doi:10.1177/0269215518818866

46. Eriksson L, Lindström B, Ekenberg L. Patients' experiences of telerehabilitation at home after shoulder joint replacement. $J$ Telemed Telecare. 2011;17(1):25-30. doi:10.1258/ jtt.2010.100317

47. García-Gómez S, Pérez-Tejero J, Hoozemans M, Barakat R. Effect of a home-based exercise program on shoulder pain and range of motion in elite wheelchair basketball players: a non-randomized controlled trial. Sports. 2019;7(8):180. doi:10.3390/sports7080180

48. Burns DM, Leung N, Hardisty M, Whyne C, Henry P, McLachlin S. Shoulder physiotherapy exercise recognition: machine learning the inertial signals from a smartwatch. Physiol Meas. 2018;39(7):075007. doi:10.1088/1361-6579/aacfd9

49. Elgert L, Steiner B, Saalfeld B, Marschollek M, Wolf K-H. Health-Enabling technologies to assist patients with musculoskeletal shoulder disorders when exercising at home: scoping review. JMIR Rehabil Assistive Tech. 2021;8(1):e21107-e21107. doi:10.2196/21107

50. Macías-Hernández SI, Vásquez-Sotelo DS, Ferruzca-Navarro $\mathrm{MV}$, et al. Proposal and evaluation of a telerehabilitation platform designed for patients with partial rotator cuff tears: a preliminary study. Ann Rehabil Med. 2016;40(4):710-717. doi:10.5535/ arm.2016.40.4.710

51. Pekyavas NO, Ergun N. Comparison of virtual reality exergaming and home exercise programs in patients with subacromial impingement syndrome and scapular dyskinesis: short term effect. Acta Orthop Traumatol Turc. 2017;51(3):238-242. doi:10.1016/j. aott.2017.03.008

52. Carbonaro N, Lucchesi I, Lorusssi F, Tognetti A Tele-monitoring and tele-rehabilitation of the shoulder muscular-skeletal diseases through wearable systems. 40th Annual International Conference of the IEEE Engineering in Medicine and Biology Society (EMBC). 2018;2018:4410-4413. doi: 10.1109/ EMBC.2018.8513371.

53. Ongvisatepaiboon K, Chan JH, Vanijja V. Smartphone-based tele-rehabilitation system for frozen shoulder using a machine learning approach. IEEW Symposium Series on Computational Intelligence. 2015;811-815.

54. Malliaras $\mathrm{P}$, Cridland $\mathrm{K}$, Hopmans $\mathrm{R}$, et al. Internet and Telerehabilitation-Delivered Management of Rotator Cuff-Related Shoulder Pain (INTEL Trial): randomized Controlled Pilot and Feasibility Trial. JMIR Mhealth Uhealth. 2020;8(11):e24311. doi:10.2196/24311

55. Van Straaten MG, Cloud BA, Morrow MM, Ludewig PM, Zhao KD. Effectiveness of home exercise on pain, function, and strength of manual wheelchair users with spinal cord injury: a high-dose shoulder program with telerehabilitation. Arch Phys Med Rehabil. 2014;95(10):1810-1817.e2. doi:10.1016/j. apmr.2014.05.004 
56. Mintken PE, Glynn P, Cleland JA. Psychometric properties of the shortened disabilities of the Arm, Shoulder, and Hand Questionnaire (QuickDASH) and Numeric Pain Rating Scale in patients with shoulder pain. J Shoulder Elbow Surg. 2009;18 (6):920-926. doi:10.1016/j.jse.2008.12.015

57. Rysstad T, Grotle M, Klokk LP, Tveter AT. Responsiveness and minimal important change of the QuickDASH and PSFS when used among patients with shoulder pain. BMC Musculoskelet Disord. 2020;21:328. doi:10.1186/s12891-020-03289-z

58. Spitzer RL, Kroenke K, Williams JB, Löwe B. A brief measure for assessing generalized anxiety disorder: the GAD-7. Arch Intern Med. 2006;166(10):1092-1097. doi:10.1001/ archinte.166.10.1092

59. Kroenke K, Spitzer RL, Williams JB. The PHQ-9: validity of a brief depression severity measure. J Gen Intern Med. 2001;16 (9):606-613. doi:10.1046/j.1525-1497.2001.016009606.x

60. Bijker L, Sleijser-Koehorst MLS, Coppieters MW, Cuijpers P, Scholten-Peeters GGM. Preferred Self-Administered Questionnaires to Assess Depression, Anxiety and Somatization in People With Musculoskeletal Pain - A Modified Delphi Study. J Pain. 2020;21(3-4):409-417. doi:10.1016/j.jpain.2019.08.006

61. George SZ, Valencia C, Beneciuk JM. A psychometric investigation of fear-avoidance model measures in patients with chronic low back pain. J Orthop Sports Phys Ther. 2010;40(4):197-205. doi:10.2519/jospt.2010.3298

62. Mintken PE, Cleland JA, Whitman JM, George SZ. Psychometric properties of the Fear-Avoidance Beliefs Questionnaire and Tampa Scale of Kinesiophobia in patients with shoulder pain. Arch Phys Med Rehabil. 2010;91(7):1128-1136. doi:10.1016/j. apmr.2010.04.009

63. Ospina M, Dennett L, Waye A, Jacobs P, Thompson A. A systematic review of the measurement properties instruments assessing presenteeism. Am J Managed Care. 2015;21(2):e17185.

64. Duncan TE, Duncan SC. An introduction to latent growth curve modeling. Behav Ther. 2004;35(2):333-363. doi:10.1016/S00057894(04)80042-X

65. Ferrer E, Hamagami F, McArdle JJ. Modeling latent growth curves with incomplete data using different types of structural equation modeling and multilevel software. Struct Equation Modeling. 2004;11(3):452-483. doi:10.1207/ s15328007sem1103_8

66. Preacher KJ, Wichman AL, MacCallum RC, Briggs NE. Latent Growth Curve Modeling. SAGE publications, Inc; 2008.

67. Kenneth AB, Curran PJ. Latent Curve Models: A Structural Equation Perspective. Wiley Series; 2006.

68. Iacobucci D. Structural equations modeling: fit Indices, sample size, and advanced topics. J Consumer Psychol. 2010;20 (1):90-98. doi:10.1016/j.jcps.2009.09.003

69. Brown TA. Confirmatory Factor Analysis for Applied Research. 2 ed. The Guilford Press; 2006.

70. Kosterink SM, Huis RM, Cagnie B, Hasenbring M, VollenbroekHutten MM. The clinical effectiveness of a myofeedback-based teletreatment service in patients with non-specific neck and shoulder pain: a randomized controlled trial. $J$ Telemed Telecare. 2010;16(6):316-321. doi:10.1258/jtt.2010.006005

71. Ingwersen KG, Jensen SL, Sørensen L, et al. Three Months of Progressive High-Load Versus Traditional Low-Load Strength Training Among Patients With Rotator Cuff Tendinopathy: primary Results From the Double-Blind Randomized Controlled RoCTEx Trial. Orthop J Sports Med. 2017;5 (8):2325967117723292. doi:10.1177/2325967117723292

72. Bennell K, Wee E, Coburn S, et al. Efficacy of standardised manual therapy and home exercise programme for chronic rotator cuff disease: randomised placebo controlled trial. BMJ. 2010;340: c2756. doi:10.1136/bmj.c2756
73. Heron SR, Woby SR, Thompson DP. Comparison of three types of exercise in the treatment of rotator cuff tendinopathy/shoulder impingement syndrome: a randomized controlled trial. Physiotherapy. 2017;103(2):167-173. doi:10.1016/j. physio.2016.09.001

74. Littlewood C, Bateman M, Brown K, et al. A self-managed single exercise programme versus usual physiotherapy treatment for rotator cuff tendinopathy: a randomised controlled trial (the SELF study). Clin Rehabil. 2016;30(7):686-696. doi:10.1177/ 0269215515593784

75. Maenhout AG, Mahieu NN, De Muynck M, De Wilde LF, Cools AM. Does adding heavy load eccentric training to rehabilitation of patients with unilateral subacromial impingement result in better outcome? A randomized, clinical trial. Knee Surg Sports Traumatol Arthrosc. 2013;21(5):1158-1167. doi:10.1007/s00167012-2012-8

76. Hopewell S, Keene DJ, Heine P, et al. Progressive exercise compared with best-practice advice, with or without corticosteroid injection, for rotator cuff disorders: the GRASP factorial RCT. Health Technol Assess. 2021;25(48):1-158. doi:10.3310/hta25480

77. Raiszadeh K, Tapicer J, Taitano L, Wu J, Shahidi B. In-Clinic Versus Web-Based Multidisciplinary Exercise-Based Rehabilitation for Treatment of Low Back Pain: prospective Clinical Trial in an Integrated Practice Unit Model. J Med Internet Res. 2021;23(3):e22548. doi:10.2196/22548

78. Garcia LM, Birckhead BJ, Krishnamurthy P, et al. An 8-Week Self-Administered At-Home Behavioral Skills-Based Virtual Reality Program for Chronic Low Back Pain: double-Blind, Randomized, Placebo-Controlled Trial Conducted During COVID-19. J Med Internet Res. 2021;23(2):e26292. doi: $10.2196 / 26292$

79. Sun E, Moshfegh J, Rishel CA, Cook CE, Goode AP, George SZ. Association of Early Physical Therapy With Long-term Opioid Use Among Opioid-Naive Patients With Musculoskeletal Pain. JAMA Network Open. 2018;1(8):e185909. doi:10.1001/ jamanetworkopen.2018.5909

80. Diercks R, Bron C, Dorrestijn O, et al. Guideline for diagnosis and treatment of subacromial pain syndrome: a multidisciplinary review by the Dutch Orthopaedic Association. Acta Orthop. 2014;85(3):314-322. doi:10.3109/17453674.2014.920991

81. Kuhn JE, Dunn WR, Sanders R, et al. Effectiveness of physical therapy in treating atraumatic full-thickness rotator cuff tears: a multicenter prospective cohort study. J Shoulder Elbow Surg. 2013;22(10):1371-1379. doi:10.1016/j.jse.2013.01.026

82. Coronado RA, Seitz AL, Pelote E, Archer KR, Jain NB. Are Psychosocial Factors Associated With Patient-reported Outcome Measures in Patients With Rotator Cuff Tears? A Systematic Review. Clin Orthop Relat Res. 2018;476(4):810-829. doi:10.1007/s11999.0000000000000087

83. Wong WK, Li MY, Yung PS, Leong HT. The effect of psychological factors on pain, function and quality of life in patients with rotator cuff tendinopathy: a systematic review. Musculoskelet Sci Pract. 2020;47:102173. doi:10.1016/j.msksp.2020.102173

84. Wolfensberger A, Vuistiner P, Konzelmann M, Plomb-Holmes C, Léger B, Luthi F. Clinician and Patient-reported Outcomes Are Associated With Psychological Factors in Patients With Chronic Shoulder Pain. Clin Orthop Relat Res. 2016;474(9):2030-2039. doi:10.1007/s11999-016-4894-0

85. Roh YH, Lee BK, Noh JH, Oh JH, Gong HS, Baek GH. Effect of depressive symptoms on perceived disability in patients with chronic shoulder pain. Arch Orthop Trauma Surg. 2012;132 (9):1251-1257. doi:10.1007/s00402-012-1545-0

86. Maestroni L, Read P, Bishop C, et al. The Benefits of Strength Training on Musculoskeletal System Health: practical Applications for Interdisciplinary Care. Sports Med. 2020;50 (8):1431-1450. doi:10.1007/s40279-020-01309-5 
87. Haik MN, Alburquerque-Sendín F, Fernandes RAS, et al. Biopsychosocial Aspects in Individuals with Acute and Chronic Rotator Cuff Related Shoulder Pain: classification Based on a Decision Tree Analysis. Diagnostics. 2020;10:11. doi:10.3390/ diagnostics 10110928

88. Chester R, Jerosch-Herold C, Lewis J, Shepstone L. Psychological factors are associated with the outcome of physiotherapy for people with shoulder pain: a multicentre longitudinal cohort study. Br J Sports Med. 2018;52(4):269-275. doi:10.1136/bjsports-2016-096084

89. van den Heuvel SG, Ijmker S, Blatter BM, de Korte EM. Loss of productivity due to neck/shoulder symptoms and hand/arm symptoms: results from the PROMO-study. J Occup Rehabil. 2007;17 (3):370-382. doi:10.1007/s10926-007-9095-y

90. Tsuji T, Matsudaira K, Sato H, Vietri J, Jaffe DH. Association between presenteeism and health-related quality of life among Japanese adults with chronic lower back pain: a retrospective observational study. BMJ Open. 2018;8(6):e021160. doi:10.1136/bmjopen-2017-021160

91. Cochrane A, Higgins NM, Rothwell C, et al. Work Outcomes in Patients Who Stay at Work Despite Musculoskeletal Pain. J Occup Rehabil. 2018;28(3):559-567. doi:10.1007/s10926-017-9748-4

92. Bennell KL, Marshall CJ, Dobson F, Kasza J, Lonsdale C, Hinman RS. Does a Web-Based Exercise Programming System Improve Home Exercise Adherence for People With Musculoskeletal Conditions?: a Randomized Controlled Trial. Am J Phys Med Rehabil. 2019;98(10):850-858. doi:10.1097/ phm.0000000000001204

93. Cranen K, Drossaert CH, Brinkman ES, Braakman-Jansen AL, Ijzerman MJ, Vollenbroek-Hutten MM. An exploration of chronic pain patients' perceptions of home telerehabilitation services. Health Expect. 2012;15(4):339-350. doi:10.1111/j.1369-7625.2011.00668.x

94. Argent R, Daly A, Caulfield B. Patient Involvement With Home-Based Exercise Programs: can Connected Health Interventions Influence Adherence? JMIR Mhealth Uhealth. 2018;6(3):e47. doi:10.2196/mhealth.8518
95. Thorpe AM, O’Sullivan PB, Mitchell T, et al. Are Psychologic Factors Associated With Shoulder Scores After Rotator Cuff Surgery? Clin Orthop Relat Res. 2018;476(10):2062-2073. doi:10.1097/CORR.0000000000000389

96. Keefe FJ, Main CJ, George SZ. Advancing Psychologically Informed Practice for Patients With Persistent Musculoskeletal Pain: promise, Pitfalls, and Solutions. Phys Ther. 2018;98 (5):398-407. doi:10.1093/ptj/pzy024

97. Slade SC, Patel S, Underwood M, Keating JL. What are patient beliefs and perceptions about exercise for nonspecific chronic low back pain? A systematic review of qualitative studies. Clin J Pain. 2014;30(11):995-1005. doi:10.1097/ ajp.0000000000000044

98. Hyphantis T, Kotsis K, Voulgari PV, Tsifetaki N, Creed F, Drosos AA. Diagnostic accuracy, internal consistency, and convergent validity of the Greek version of the patient health questionnaire 9 in diagnosing depression in rheumatologic disorders. Arthritis Care Res. 2011;63(9):1313-1321. doi:10.1002/acr.20505

99. Jack K, McLean SM, Moffett JK, Gardiner E. Barriers to treatment adherence in physiotherapy outpatient clinics: a systematic review. Man Ther. 2010;15(3):220-228. doi:10.1016/j. math.2009.12.004

100. Weber S, Chahal J. Management of Rotator Cuff Injuries. AAOS Clinical Practice Guideline Summary. J Am Acad Orthopaedic Surgeons. 2019;28(5):e193-201. doi:10.5435/JAAOS-D-1900463

101. Seitz AL, Mcclure PW, Finucane S, Boardman ND, Michener LA. Mechanisms of rotator cuff tendinopathy: intrinsic, extrinsic, or both? Clin Biomech. 2011;26(1):1-12. doi:10.1016/j. clinbiomech.2010.08.001
Journal of Pain Research

\section{Publish your work in this journal}

The Journal of Pain Research is an international, peer reviewed, open access, online journal that welcomes laboratory and clinical findings in the fields of pain research and the prevention and management of pain. Original research, reviews, symposium reports, hypothesis formation and commentaries are all considered for publication. The manuscript

\section{Dovepress}

management system is completely online and includes a very quick and fair peer-review system, which is all easy to use. Visit http:// www.dovepress.com/testimonials.php to read real quotes from published authors. 